Désirée Y. Visser

Nicolaas J. Jansen

Marloes M. Ijland

Tom J. de Koning

Peter M. van Hasselt

\title{
Intracranial bleeding due to vitamin $K$ deficiency: advantages of using a pediatric intensive care registry
}

Received: 9 July 2010

Accepted: 30 January 2011

Published online: 11 March 2011

(C) The Author(s) 2011. This article is published with open access at

Springerlink.com

D. Y. Visser - T. J. de Koning .

P. M. van Hasselt ( $\bullet$ )

Department of Pediatric Metabolic

Diseases, Wilhelmina Children's Hospital,

University Medical Center Utrecht,

Room KC 03.063.0, PO Box 85090,

3508 AB Utrecht, The Netherlands

e-mail: p.vanhasselt@umcutrecht.nl

Tel.: +31-88-7554003

Fax: $+31-88-7555350$

\section{N. J. Jansen}

Department of Pediatric Intensive Care,

Wilhelmina Children's Hospital, University

Medical Center Utrecht, Utrecht,

The Netherlands

M. M. Ijland

Department of Pediatric Intensive Care, Radboud University Nijmegen Medical Center, Nijmegen, The Netherlands
Abstract Aim: To determine the incidence of late intracranial vitamin $\mathrm{K}$ deficiency bleeding (VKDB) in The Netherlands using the Dutch Pediatric Intensive Care Evaluation (PICE) registry. Methods: The PICE registry was used to identify all infants who were admitted to a Dutch pediatric intensive care unit (PICU) with intracranial bleeding between 1 January 2004 and 31 December 2007. Cases of confirmed late intracranial VKDB were used to calculate the incidence for each year. To estimate the completeness of ascertainment of the PICE registry, data from 2005 were compared with general surveillance data from that year.

Results: In the 4-year study period, $16 / 64(25 \%)$ of the infants admitted with intracranial bleeding had late intracranial VKDB, resulting in an overall incidence of 2.1/100,000 live births $(95 \%$ confidence interval 1.2-3.5). The single-year incidence varied markedly between 0.5 and 3.3 per 100,000 live births. All five ascertained cases in 2005 were identified using the PICE registry, while general surveillance identified only three. Conclusions: The PICE registry allows ongoing monitoring of the incidence of late intracranial VKDB and appears to be associated with a higher rate of completeness than general surveillance. We propose the use of pediatric intensive care registries to assess the efficacy of national vitamin $\mathrm{K}$ prophylactic regimens.

Keywords Vitamin K deficiency . Intracranial bleeding · Prophylaxis . General surveillance .

Pediatric Intensive Care Evaluation registry

\section{Introduction}

Intracranial hemorrhage is a frequent manifestation of vitamin $\mathrm{K}$ deficiency (VKD) in infancy. It is the presenting symptom in approximately $50 \%$ of cases with late vitamin $\mathrm{K}$ deficiency bleeding (VKDB) in Western Europe, with even higher rates (up to $82 \%$ ) reported in developing countries [1-10]. As such intracranial bleeding is associated with a high mortality rate and serious neurological sequelae [1, 3-6, 11-13], preventing it is a major objective of national vitamin $\mathrm{K}$ prophylactic regimens worldwide.
Thus far, the efficacy of vitamin $\mathrm{K}$ prophylactic regimens is evaluated on the basis of the incidence of VKDB under a given regimen. The incidence is calculated from the number of ascertained cases from general surveillance studies. However, as illustrated by two surveillance studies from The Netherlands, these data should be interpreted with caution. Both a low incidence of 1.1/ 100,000 (in 1992-1994) and a relatively high incidence of $3.2 / 100,000$ (in 2005) were reported using this methodology under a single prophylactic regimen [3, 14]. These data underline that the incidence is not merely a reflection 
of the efficacy of a prophylactic regimen. Random variations in factors strongly associated with VKDBparticularly the rate of breastfeeding and the incidence of cholestasis-may have a significant impact on the incidence. In addition, the degree of completeness of ascertainment may play a pivotal role. Particularly, a low degree of completeness will cause underestimation of the incidence of late VKDB and hence false reassurance regarding the effectiveness of prophylaxis.

Alternative approaches may overcome the drawbacks of general surveillance. We previously showed that registries for cholestatic liver diseases can be used to determine the protection offered to high-risk infants [9, 15]. Here, based on the observation that infants with late intracranial $\mathrm{VKDB}$ are routinely admitted to a pediatric intensive care unit (PICU), we investigated whether a pediatric intensive care registry can be used to more reliably determine the incidence of late intracranial VKDB. Retrieving cases of late intracranial VKDB from the Dutch pediatric intensive care registry over a 4-year timeframe not only allowed a view on the year-to-year variation of late intracranial VKDB, but also allowed us to compare its completeness with general surveillance.

\section{Methods}

Patients

The diagnoses of all infants admitted to the eight Dutch PICUs are registered in the National Pediatric Intensive Care Evaluation (PICE) registry from 2003 onwards. The PICE registry was used to identify infants between the age of 8 days and 6 months, who were admitted to a Dutch PICU with intracranial bleeding between 1 January 2004 and 31 December 2007. To minimize the risk of missing infants with late intracranial VKDB, the search strategy was designed to allow detection through the diagnosis intracranial bleeding, through the symptoms of intracranial bleeding, and through the underlying disorder. Search items were: brain dead, cerebral infarct or stroke, intracranial hemorrhage, convulsions, meningitis, gastrointestinal bleeding, hepatitis, other liver diseases, biliary atresia, neonatal jaundice, other gastrointestinal diseases, and coagulation defects. Of the infants who were admitted to a PICU with intracranial bleeding, discharge letters and laboratory data were studied to determine the causes of the intracranial bleeding. Patient files of infants suspected of intracranial VKDB were reviewed to establish whether VKD was present and to obtain relevant clinical characteristics.

Approval for the study was obtained from the University Medical Center Utrecht Ethics Committee.
Late intracranial VKDB

Late intracranial VKDB was defined as intracranial bleeding (confirmed using computer tomography or magnetic resonance imaging) in the presence of prothrombin ratio $(P R) \geq 4$ which normalized after administration of vitamin $K$, in an infant between the age of 8 days and 6 months [8,9]. If determined, the international normalized ratio (INR) was used as PR. If only a prothrombin time (PT) in seconds was available, the PR was calculated with the formula: $\mathrm{PR}=\mathrm{PT}_{\text {patient }} / \mathrm{PT}_{\text {control }}$. If $\mathrm{PT}_{\text {control }}$ was not determined, the mean of the provided reference rate was used as $\mathrm{PT}_{\text {control }}$ [9]. If available, the concentration of proteins induced by vitamin $\mathrm{K}$ absence (PIVKAs) had to be above the reference value of the used assay [8].

\section{Clinical characteristics}

Of the infants with late intracranial VKDB, the values of several biochemical parameters and the age and weight at diagnosis were obtained, as well as information concerning the severity, outcome, and etiology of the bleeding. Age at diagnosis was defined as the age of the infant when first seen by a doctor with complaints related to VKD. Cholestasis was defined as total serum bilirubin concentration $>50 \mu \mathrm{mol} / 1$ with direct fraction $\geq 20 \%$ [9]. When administration of vitamin $\mathrm{K}$ prophylaxis to an infant was mentioned in the patient file but the quantity of vitamin $\mathrm{K}$ was not specified, it was assumed that the Dutch vitamin $\mathrm{K}$ prophylactic regimen was followed, consisting of administration of $1 \mathrm{mg}$ vitamin $\mathrm{K}$ orally directly after birth followed by daily administration of $25 \mu \mathrm{g}$ vitamin $\mathrm{K}$ orally from 1 week to 3 months post partum [16]. According to the absence or presence of an underlying disorder predisposing to $\mathrm{VKD}$, late intracranial VKDB was subclassified as idiopathic late intracranial VKDB or secondary late intracranial VKDB, respectively [8].

\section{Statistical analysis}

To determine the overall incidence of late intracranial VKDB and to assess completeness of ascertainment of late intracranial VKDB, a capture-recapture analysis was performed on the patients captured in 2005 by the PICE registry and general surveillance [3]. Birth date, gender, and initials from patients captured by the PICE registry were retrieved from local databases and were used to identify the infants detected in both studies. The total number of infants with late intracranial VKDB in 2005 was calculated with the formula [17] 
Table 1 Incidence of intracranial bleeding and late intracranial VKDB in The Netherlands calculated by using the PICE registry

\begin{tabular}{llllll}
\hline & 2004 & 2005 & 2006 & 2007 & $2004-2007$ \\
\hline Live births $(N)$ & 194,007 & 187,910 & 185,057 & 181,336 & 748,310 \\
Patients admitted to a PICU $(N)$ & 4,532 & 4,663 & 4,612 & 4,426 & 18,233 \\
Intracranial bleeding $(N)$ & 18 & 21 & 9 & 16 & 64 \\
Incidence of intracranial bleeding & $9.3 / 100,000$ & $11.2 / 100,000$ & $4.9 / 100,000$ & $8.8 / 100,000$ & $8.6 / 100,000$ \\
$\quad(95 \%$ CI) & $(5.5-14.7)$ & $(6.9-17.1)$ & $(2.2-9.2)$ & $(5.0-14.3)$ & $(6.6-11.0)$ \\
Late intracranial VKDB $(N)$ & 4 & 5 & 1 & 6 & 16 \\
Incidence of late intracranial VKDB & $2.1 / 100,000$ & $2.7 / 100,000$ & $0.5 / 100,000$ & $3.3 / 100,000$ & $2.1 / 100,000$ \\
$(95 \%$ CI) & $(0.6-5.3)$ & $(0.9-6.2)$ & $(0.0-3.0)$ & $(1.2-7.2)$ & $(1.2-3.5)$ \\
\hline
\end{tabular}

$V K D B$ vitamin $\mathrm{K}$ deficiency bleeding, $P I C E$ pediatric intensive care evaluation, $N$ number, $P I C U$ pediatric intensive care unit, $95 \% C I$ 95\% confidence interval

${ }^{\mathrm{a}}$ Incidence calculated per 100,000 live births

$$
N=\frac{\left(n_{1}+1\right)\left(n_{2}+1\right)}{\left(n_{12}+1\right)}-1
$$

In this formula $N$ is the estimated total number of infants with late intracranial VKDB, $n_{1}$ is the number of infants with late intracranial VKDB detected with the PICE registry, $n_{2}$ is the number of infants with late intracranial VKDB detected with general surveillance, and $n_{12}$ is the number of infants with late intracranial VKDB who were detected in both studies. The 95\% confidence interval was calculated with the formula [18]

$$
N \pm 1.96 \sqrt{\frac{\left(n_{1}+1\right)\left(n_{2}+1\right)\left(n_{1}-n_{12}\right)\left(n_{2}-n_{12}\right)}{\left(n_{12}+1\right)^{2}\left(n_{12}+2\right)}} .
$$

The total number of infants with late intracranial VKDB in 2005 was used to determine the incidence of late intracranial VKDB in that year. The $95 \%$ confidence interval of the incidence was calculated according to a Poisson distribution. Completeness of a surveillance strategy was defined as the percentage of the calculated total of infants with late intracranial VKDB retrieved by the respective strategy.

\section{Results}

Intracranial bleeding

Between 1 January 2004 and 31 December 2007, 18,233 infants were admitted to the Dutch PICUs. The search strategy retrieved 428 infants, 64 of whom were diagnosed with intracranial bleeding (Table 1). Child abuse was the proven (5 patients) or highly suspected (12 patients) cause of intracranial bleeding in 17 patients. Sixteen infants had confirmed late intracranial VKDB (Table 2). In one additional infant, late intracranial VKDB was suspected, but could not be confirmed, because vitamin $\mathrm{K}$ was already supplemented before the coagulation factors were measured and no PIVKA
Table 2 Causes of intracranial bleeding in infants between the age of 8 days and 6 months admitted to a Dutch PICU between 2004 and 2007

\begin{tabular}{lcc}
\hline Cause & Number & $\begin{array}{l}\text { Percentage } \\
(\%)\end{array}$ \\
\hline Child abuse & 17 & 26.6 \\
Vitamin K deficiency & 17 & 26.6 \\
Confirmed & 16 & 25.0 \\
Suspected & 1 & 1.6 \\
Accidental head trauma & 10 & 15.6 \\
Other coagulation disorders & 6 & 9.4 \\
Congenital cardiovascular abnormality & 4 & 6.3 \\
Iatrogenous & 3 & 4.7 \\
Vascular malformation & 2 & 3.1 \\
Intraventricular bleeding due to prematurity & 2 & 3.1 \\
Unknown & 2 & 3.1 \\
Tumor & 1 & 1.6 \\
Total & 64 & 100 \\
\hline
\end{tabular}

PICU pediatric intensive care unit

analysis was performed. Mean age at presentation in patients with late intracranial VKDB was significantly lower than in patients with intracranial bleeding due to child abuse (47 versus 90 days, $p=0.01$ ) (Fig. 1). As a consequence, VKD was the most common cause of intracranial bleeding in the first 3 months of life (14/41, $34 \%)$.

\section{Late intracranial VKDB}

Of the 16 infants with confirmed late intracranial VKDB, 12 were boys $(75 \%)$. Mean age at diagnosis was 47 days (Table 3). In eight infants (50\%) so-called warning bleeds (e.g., hematemesis, nasal bleeding, and ecchymoses) were seen before symptoms consistent with late intracranial VKDB occurred. The most common locations of the intracranial VKDB were subdural $(8 / 16,50 \%)$ and intracerebral $(6 / 16,38 \%)$. Eleven infants $(69 \%)$ required mechanical ventilation. Seven infants $(44 \%)$ underwent neurosurgical intervention. In three additional infants 


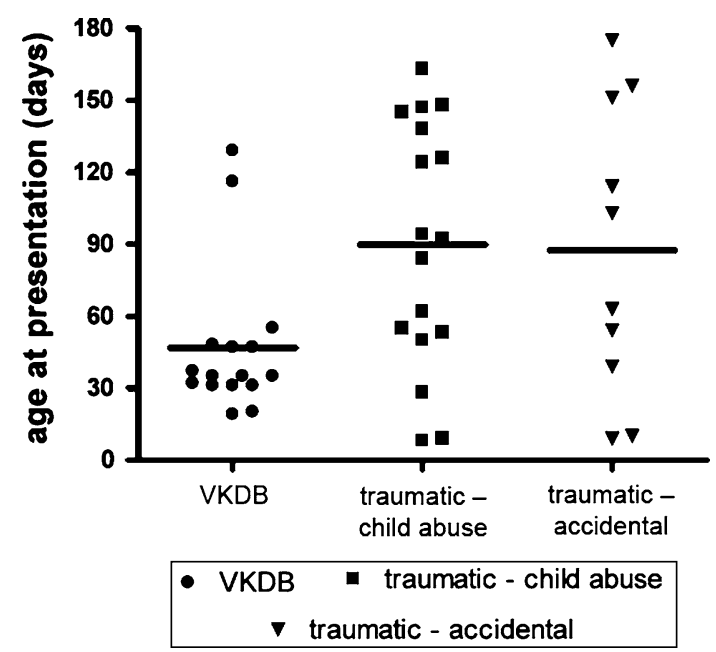

Fig. 1 Comparison of mean age at presentation in patients with intracranial bleeding due to vitamin $\mathrm{K}$ deficiency, child abuse, and accidental head trauma. $V K D B$ vitamin $\mathrm{K}$ deficiency bleeding

Table 3 Characteristics of infants with confirmed late intracranial VKDB admitted to a Dutch PICU between 2004 and 2007

Feature

Male/female, $N(\%)$

Birth weight, mean (range), $\mathrm{g}$

Age at diagnosis, mean (range), days

Weight at diagnosis, mean (range), $g$

Biochemical parameters

Bilirubin total, median (range), $\mu \mathrm{mol} / \mathrm{l}$

Bilirubin direct, median (range), $\mu \mathrm{mol} / 1$

ASAT, median (range), U/l

ALAT, median (range), U/l

Etiology

Cholestasis, $N(\%)$

Underlying disorder, $N(\%)$

Breastfeeding, $N(\%)$

Severity of bleeding

Mechanical ventilation, $N(\%)$

Neurosurgical intervention, $N(\%)$

No neurosurgical intervention due to poor condition, $N(\%)$

Outcome

Died, $N(\%)$

Neurological sequelae, $N(\%)$
$12(75) / 4(25)$

$3,339(2,160-4,010)$

$47(19-129)$

4,178 (3,200-5,240)

$82(8-226)$

$52(5-134)$

$92(33-773)$

48 (20-869)

$13(81)$

$14(88)$

$14(88)$

11 (69)

7 (44)

3 (19)

$6(38)$

$6(38)$
$V K D B$ vitamin $\mathrm{K}$ deficiency bleeding, $P I C U$ pediatric intensive care unit, $N$ number, $A S A T$ asparagine aminotransferase, $A L A T$ alanine aminotransferase

(19\%), a neurosurgical intervention was considered but could not be performed because of the poor condition of the infant. In total, six patients died as a consequence of intracranial bleeding (38\%) and six infants had severe neurological sequelae after a follow-up period of 6 months (38\%).

Fourteen of the 16 infants had secondary late intracranial VKDB (88\%), of which 13 were due to cholestasis $(81 \%)$ (Table 4). Although no disorder predisposing to
VKD could be established in two cases, abnormalities consistent with fat malabsorption were present in both patients. One infant had elevated serum bile acids $(22 \mu \mathrm{mol} / \mathrm{l})$, indicative of cholestasis. The other infant had diminished values of cholesterol and triglycerides (1.0 and $<0.1 \mathrm{mmol} / \mathrm{l}$, respectively). Cystic fibrosis was considered but excluded in this patient. Fourteen infants were fully breastfed (88\%). At least 10/14 (71\%) had received the recommended vitamin $\mathrm{K}$ prophylaxis. In two infants the degree of adherence could not be retrieved. In the two additional infants adherence to prophylaxis was suboptimal; one infant did not receive the $1 \mathrm{mg}$ vita$\min \mathrm{K}$ on the first day post partum, and the other infant only received $25 \mu \mathrm{g}$ vitamin $\mathrm{K}$ weekly after the $1 \mathrm{mg}$ vitamin $\mathrm{K}$ post partum.

\section{Incidence of late intracranial VKDB}

Between 2004 and 2007 the annual incidence of late intracranial VKDB varied from 0.5 per 100,000 live births (95\% CI: $0.0-3.0)$ to 3.3 per 100,000 live births (95\% CI: 1.2-7.2) (Table 1). The average incidence of late intracranial VKDB over 2004-2007 was 2.1 per 100,000 live births (95\% CI: 1.2-3.5).

\section{Completeness of ascertainment}

To evaluate completeness we compared the cases of late intracranial VKDB in 2005 identified using the PICE registry with those identified using general surveillance. Five infants with late intracranial VKDB were identified by the PICE registry, compared with three infants (out of six with VKDB) identified through general surveillance [3]. As the latter infants were also identified by the PICE registry, the estimated total number of infants with late intracranial VKDB in 2005 was five. The characteristics and severity of the two cases missed using general surveillance did not differ from the characteristics and severity of the other three cases.

\section{Discussion}

This is the first study to demonstrate that a pediatric intensive care registry is a novel instrument to determine the incidence of late intracranial VKDB. This strategy allows long-term monitoring of late intracranial VKDB and results in a higher degree of completeness than general surveillance studies. Furthermore these data show that VKD - a preventable disease-is the most frequent cause of intracranial bleeding in the first 3 months of life in infants admitted to Dutch pediatric intensive care units. 


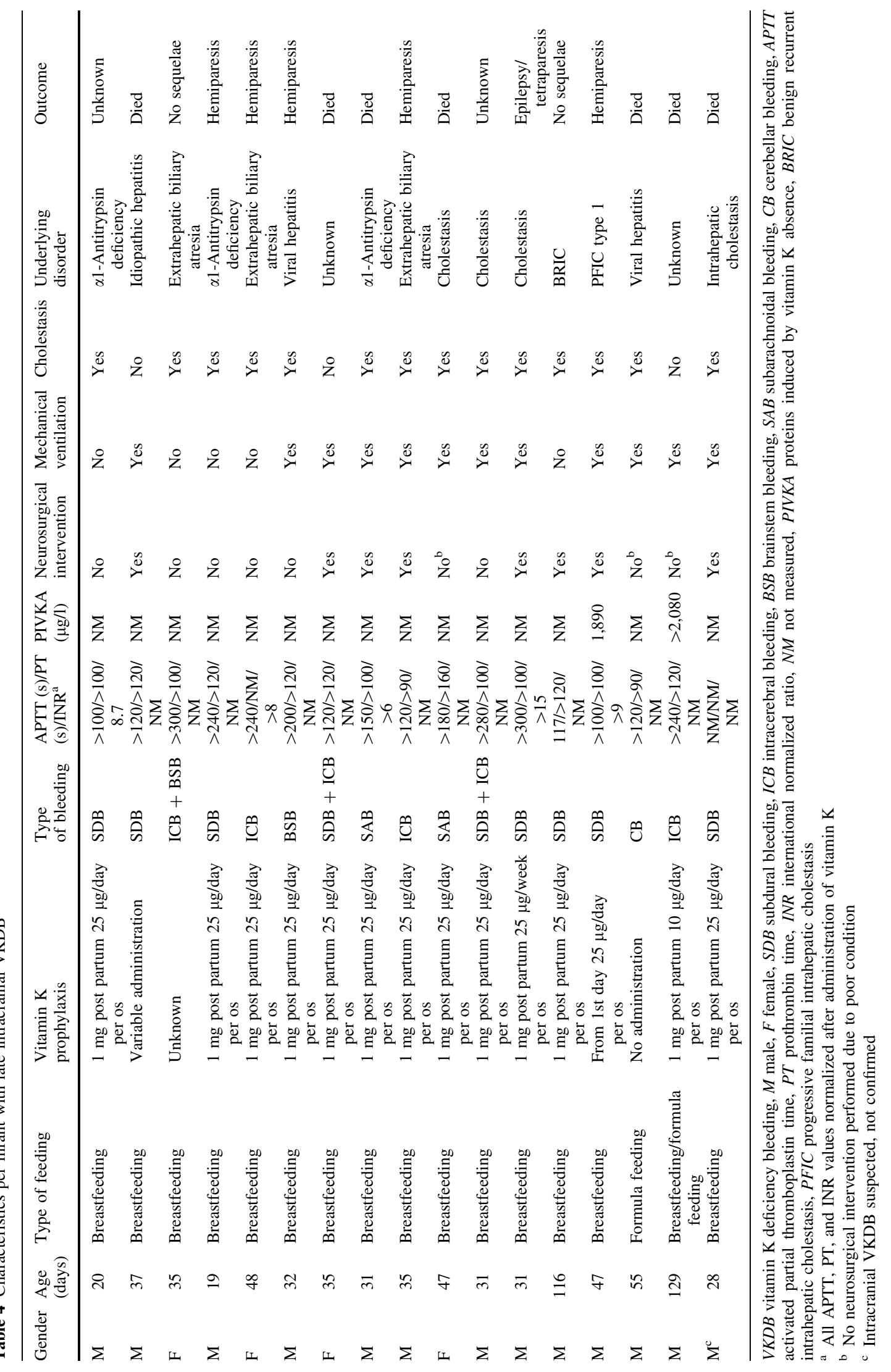


Previously, Danielson et al. [19] identified patients with late intracranial VKDB by retrieving patient files of intracranial hemorrhages. Applying this approach to the pediatric intensive care registry provided a dataset with the same nationwide coverage as that obtained using general surveillance, a prerequisite for performing capture-recapture analysis. This analysis-for the first time-allowed a view on the (lack of) completeness of general surveillance. In the year of comparison between the two methods, two out of five cases of late intracranial VKDB would have been missed using general surveillance only. Furthermore, the PICE registry data allowed us to delineate the substantial year-to-year variation in the incidence of late intracranial VKDB (a factor of 6 between the highest and lowest year incidence), confirming our suspicion that single-year surveillance may lead to erroneous conclusions. Had general surveillance been performed in 2006, the incidence of late intracranial VKDB would have been $\sim 5$ times lower than that in 2005. Finally, the data on the relative frequency of late intracranial VKDB as compared with other causes of intracranial bleeding in infancy provided a meaningful context, putting the incidence figures $(2.1 / 100,000)$ in perspective.

There are some limitations of this study. First, although completeness was higher than from general surveillance and despite all efforts to minimize this, the registry can occasionally be incomplete. In addition, cases will be missed if late intracranial VKDB causes death before admittance to a PICU or if PICU admittance is unnecessary in case of mild late intracranial VKDB. As a consequence, the real incidence of late intracranial VKDB is likely even higher than reported here. Furthermore, it should not be forgotten that an unknown number of cases of VKDB will inevitably be unnoticed because bleeding occurred at another site.

Pediatric intensive care registries are operational in various countries, including the USA (Virtual Pediatric Intensive Care Unit Performance System) and the UK (Paediatric Intensive Care Audit Network), to compare and improve the quality of care of PICUs [20]. This study exemplifies that these registries can also be used for epidemiological studies on (rare) diseases which necessitate intensive care treatment, including late intracranial VKDB. Moreover, we believe that pediatric intensive care registries can be used to compare the efficacies of different vitamin $\mathrm{K}$ prophylactic regimens and to optimize vitamin $\mathrm{K}$ prophylactic regimens.

Our data confirm that most (if not all) infants with late intracranial VKDB under the current vitamin K prophylaxis had evidence of fat malabsorption at the time of diagnosis, mostly cholestasis [3]. Notably, biliary atresia accounted for only $19 \%$ of all cases with late intracranial VKDB, much less than the $66 \%$ observed from recent general surveillance data [3]. Most other causes of cholestasis observed in this study were of more transient nature. Despite aggressive intervention, late intracranial VKDB is associated with a high rate of mortality and neurological sequelae, similar to previous reports $[1,4-6$, $11,12,21]$. The importance of recognizing the prime warning signs for late intracranial VKDB in infants (both cholestatic jaundice and seemingly harmless bleeds, e.g., hematemesis, nasal bleeding, and ecchymoses) cannot be stressed enough. However, recent data demonstrate that increased awareness of these signs alone is not sufficient to prevent VKDB [15]. Only an adequate specific vitamin $\mathrm{K}$ dosing regimen is capable of preventing such bleeding in virtually all infants $[9,22]$.

Our data underline the conclusions from general surveillance and from at-risk populations that the current Dutch vitamin $\mathrm{K}$ prophylaxis is inadequate $[3,9]$. The high frequency of late intracranial VKDB in infants and the severity of the intracranial VKDB reported here underscore the need to change the current regimen.

In conclusion, this study provides proof of principle for the use of national PICU registries for ongoing, highquality monitoring of the incidence of late intracranial vitamin $\mathrm{K}$ deficiency bleeding. In our opinion, PICU registries may provide a valuable instrument to evaluate the efficacy of vitamin K prophylactic regimens.

Acknowledgments The authors would like to thank Dr. I. Visser, MA, MSc and researcher of the Dutch Pediatric Intensive Care Evaluation registry, for his help in obtaining the study data. The authors also thank all staff members of the PICUs in The Netherlands, without whose effort data registration and thereby this work would not have been possible.

Open Access This article is distributed under the terms of the Creative Commons Attribution Noncommercial License which permits any noncommercial use, distribution, and reproduction in any medium, provided the original author(s) and source are credited.

\section{References}

1. Chuansumrit A, Isarangkura $P$, Hathirat P (1998) Vitamin K deficiency bleeding in Thailand: a 32-year history. Southeast Asian J Trop Med Public Health 29:649-654
2. Cornelissen M, von Kries R, Loughnan P, Schubiger G (1997) Prevention of vitamin $K$ deficiency bleeding: efficacy of different multiple oral dose schedules of vitamin K. Eur J Pediatr 156:126-130
3. IJland MM, Pereira RR, Cornelissen EA (2008) Incidence of late vitamin K deficiency bleeding in newborns in the Netherlands in 2005: evaluation of the current guideline. Eur J Pediatr 167:165-169 
4. McMillan DD, Grenier D, Medaglia A (2004) Canadian Paediatric Surveillance Program confirms low incidence of hemorrhagic disease of the newborn in Canada. Paediatr Child Health 9:235-238

5. McNinch A, Busfield A, Tripp J (2007) Vitamin K deficiency bleeding in Great Britain and Ireland: British Paediatric Surveillance Unit Surveys, 1993-94 and 2001-02. Arch Dis Child 92:759-766

6. Schubiger G, Berger TM, Weber R, Banziger O, Laubscher B (2003) Prevention of vitamin $\mathrm{K}$ deficiency bleeding with oral mixed micellar phylloquinone: results of a 6-year surveillance in Switzerland. Eur J Pediatr 162:885-888

7. Sutor AH, Dagres N, Niederhoff H (1995) Late form of vitamin K deficiency bleeding in Germany. Klin Padiatr 207:89-97

8. Sutor $\mathrm{AH}$, von Kries R, Cornelissen EA, McNinch AW, Andrew M (1999) Vitamin K deficiency bleeding (VKDB) in infancy. ISTH pediatric/perinatal subcommittee. International society on thrombosis and haemostasis. Thromb Haemost 81:456-461

9. van Hasselt PM, de Koning TJ, Kvist N, de Vries E, Lundin CR, Berger R, Kimpen JL, Houwen RH, Jorgensen MH, Verkade HJ (2008) Prevention of vitamin $\mathrm{K}$ deficiency bleeding in breastfed infants: lessons from the Dutch and Danish biliary atresia registries. Pediatrics 121:e857-e863
10. von Kries R, Hachmeister A, Gobel U (2003) Oral mixed micellar vitamin K for prevention of late vitamin $\mathrm{K}$ deficiency bleeding. Arch Dis Child Fetal Neonatal Ed 88:F109-F112

11. Cekinmez M, Cemil T, Cekinmez EK, Altinors N (2008) Intracranial hemorrhages due to late-type vitamin $\mathrm{K}$ deficiency bleeding. Childs Nerv Syst 24:821-825

12. Chaou WT, Chou ML, Eitzman DV (1984) Intracranial hemorrhage and vitamin $\mathrm{K}$ deficiency in early infancy. J Pediatr 105:880-884

13. Lane PA, Hathaway WE (1985) Vitamin $\mathrm{K}$ in infancy. J Pediatr 106:351-359

14. Cornelissen EA, Hirasing RA, Monnens LA (1996) Prevalence of hemorrhages due to vitamin $\mathrm{K}$ deficiency in The Netherlands, 1992-1994. Ned Tijdschr Geneeskd 140:935-937

15. van Hasselt PM, Kok K, Vorselaars $A D$, van Vlerken L, Nieuwenhuys E, de Koning TJ, de Vries RA, Houwen RH (2009) Vitamin K deficiency bleeding in cholestatic infants with alpha-1antitrypsin deficiency. Arch Dis Child Fetal Neonatal Ed 94:F456-F460

16. Uitentuis J (1990) Administration of vitamin $\mathrm{K}$ to neonates and infants. Ned Tijdschr Geneeskd 134:1642-1646

17. Chapman DG (1951) Some properties of the hypergeometric distribution with applications to zoological censuses. Univ Calif Public Stat 1:131-160
18. Papoz L, Balkau B, Lellouch J (1996) Case counting in epidemiology: limitations of methods based on multiple data sources. Int $\mathbf{J}$ Epidemiol 25:474-478

19. Danielsson N, Hoa DP, Thang NV, Vos T, Loughnan PM (2004) Intracranial haemorrhage due to late onset vitamin $\mathrm{K}$ deficiency bleeding in Hanoi province, Vietnam. Arch Dis Child Fetal Neonatal Ed 89:F546-F550

20. LaRovere JM, Jeffries HE, Sachdeva RC, Rice TB, Wetzel RC, Cooper DS, Bird GL, Ghanayem NS, Checchia PA, Chang AC, Wessel DL (2008)

Databases for assessing the outcomes of the treatment of patients with congenital and paediatric cardiac disease- the perspective of critical care. Cardiol Young 18(Suppl 2):130-136

21. Akiyama H, Okamura Y, Nagashima T, Yokoi A, Muraji T, Uetani Y (2006) Intracranial hemorrhage and vitamin $\mathrm{K}$ deficiency associated with biliary atresia: summary of 15 cases and review of the literature. Pediatr Neurosurg 42:362-367

22. Hansen KN, Minousis M, Ebbesen F (2003) Weekly oral vitamin K prophylaxis in Denmark. Acta Paediatr 92:802-805 